\title{
Enhanced Vibrational Broadening of Core-Level Photoemission from the Surface of Na(110)
}

\author{
D. M. Riffe, (a) G. K. Wertheim, and P. H. Citrin \\ AT\&T Bell Laboratories, Murray Hill, New Jersey 07974 \\ (Received 10 December 1990)
}

\begin{abstract}
High-resolution temperature-dependent photoemission data from $\mathrm{Na} 2 p$ core levels reveal substantially larger phonon broadening in the first atomic layer of $\mathrm{Na}(110)$ than in the bulk. We show that the enhanced width is due primarily to the excitation of relatively soft phonon modes perpendicular to the surface. Soft surface-phonon modes also account for previously reported but uninterpreted broadening of transition-metal surface-atom core levels.
\end{abstract}

PACS numbers: $79.60 . \mathrm{Cn}$

The coupling of vibrations to electronic transitions is manifest in a variety of solid-state systems, ranging from the classic example of optical excitation of $F$-center defects $^{1}$ to $x$-ray photoemission of core electrons in bulk insulators $^{2}$ and metals. ${ }^{3}$ More recently, core-level photoemission from atoms and molecules adsorbed on the surface have been shown to exhibit similar effects, but here the vibronic coupling is to the vibrational modes of the adsorbate-substrate bond ${ }^{4}$ rather than to those of the bulk solid. The intriguing question naturally arises regarding the nature of the vibronic coupling of atoms within the first atomic layer of a solid. Although surface-atom core-level widths exceeding bulk values have indeed been reported in several systems, the magnitude of the extra width has generally been small, and its origin, which could lie in the core-hole lifetime, phonons, or sample inhomogeneities, has generally been obscure.

Here, we report on surface-sensitive core-level photoemission from the $2 p$ levels of a (110)-oriented crystal of $\mathrm{Na}$ metal. The high-resolution spectra, collected at temperatures between 77 and $250 \mathrm{~K}$, conclusively demonstrate a substantially enhanced vibrational broadening for atoms at the surface relative to those in the bulk. This extra width is argued to arise from the relaxation of the core-excited atom normal to the surface, resulting in the excitation of relatively soft surface-phonon modes polarized in that direction. The procedures used here to isolate the contributions of phonons in the surface corelevel data, as well as our conclusions regarding the nature of the enhanced broadening itself, are generally applicable to a wide range of systems.

Data were obtained on beam line U4A at the National Synchrotron Light Source using photon energies in the range of 37.5 to $140 \mathrm{eV}$. Photoelectrons were collected with a $100-\mathrm{mm}$ VSW hemispherical analyzer operated at $40 \mathrm{meV}$ resolution, giving a total instrumental resolution between 70 and $100 \mathrm{meV}$, depending upon the photon energy. Sodium samples were grown on a $\mathrm{Ni}(100)$ substrate at $77 \mathrm{~K}$ by deposition from commercial dispensers. As determined with low-energy electron diffraction (LEED), the freshly deposited samples exhibited (110) surface orientation of several domains. Inhomogeneous broadening from atoms at domain boundaries ${ }^{5}$ was minimized by annealing the samples to $\sim 200 \mathrm{~K}$ and cooling back down to $77 \mathrm{~K}$. This resulted in more highly ordered, single-domain surfaces with significantly sharper LEED patterns and somewhat sharper core-level spectra. The temperature-dependent data were taken with $45-\mathrm{eV}$ photons as the sample warmed up continuously after turning off the liquid- $\mathrm{N}_{2}$ cooling. The temperature rise during the acquisition of a single 200 -sec spectrum was typically $5 \mathrm{~K}$. At the end of a temperature run, comprising $\sim 25$ spectra, the sample was again cooled to $77 \mathrm{~K}$ to check for changes in the spectrum due to contamination from the ambient vacuum of $\sim 1 \times 10^{-10}$ Torr. No such changes were observed.

A $\mathrm{Na} 2 p$ spectrum from a single-domain sample is shown in the upper part of Fig. 1. The three distinct

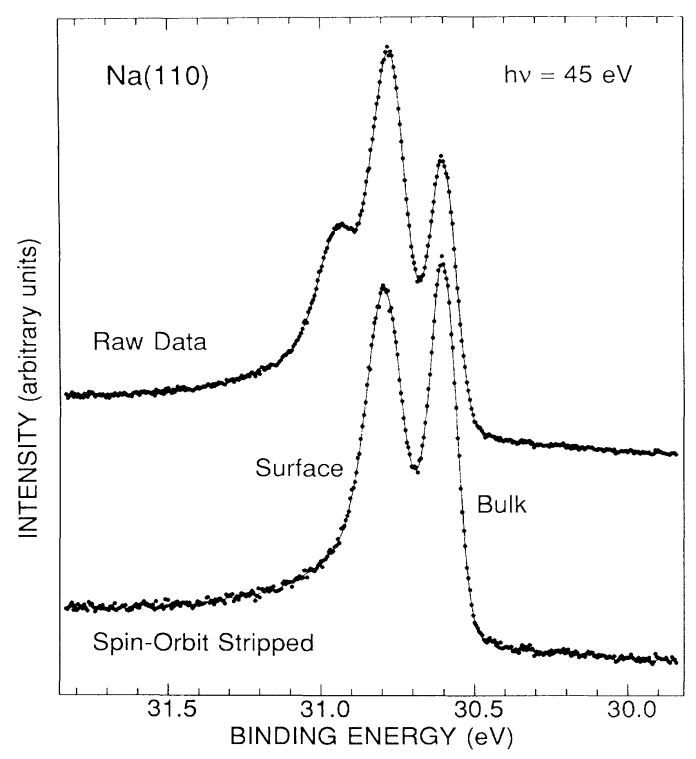

FIG. 1. Top: Raw data of $2 p$ core-level photoemission from $\mathrm{Na}(110)$ obtained at $T=80 \mathrm{~K}$. Bottom: Spin-orbit-stripped data showing only the $2 p_{3 / 2}$ component. Solid lines are smoothed representations of the data. 
peaks observed are the result of two overlapping spinorbit doublets, one from bulk and one from surface atoms. The well-resolved peak at $30.6 \mathrm{eV}$ is the bulk $2 p_{3 / 2}$ component and that at $30.95 \mathrm{eV}$ is the surface $2 p_{1 / 2}$ component. The feature between these peaks contains the unresolved surface $2 p_{3 / 2}$ and bulk $2 p_{1 / 2}$ lines.

Apart from the qualitative observation that the $2 p_{1 / 2}$ surface component is broader than the $2 p_{3 / 2}$ bulk line, quantitative information can be obtained only from more detailed analysis. A straightforward approach is to apply a subtractive stripping technique to remove the contribution of the $2 p_{1 / 2}$ components. This procedure involves no assumptions about the line shape and requires only the spin-orbit splitting and intensity ratio. We obtain satisfactory results for splittings between 155 and $165 \mathrm{meV}$ and ratios between 0.48 and 0.58 . Below the raw data in Fig. 1 we show a stripped version of the same spectrum using a splitting of $160 \mathrm{meV}$ and a ratio of 0.52 .

Several conclusions can be drawn from the stripped spectrum. First, the success of the procedure (as seen by the lack of oscillations in the tail at high binding energy) indicates that the lifetime width of the $2 p_{1 / 2}$ component is not greatly altered by the $L_{2} L_{3} M_{1}$ Coster-Kronig decay process. Second, the spin-orbit parameters of the bulk and surface components are identical, rather than different as reported in an analysis of polycrystalline $\mathrm{Na}$ data. $^{5}$ Third, the surface-atom core-level shift is 190 $\mathrm{meV}$, a value $30 \mathrm{meV}$ smaller than the polycrystalline average. 5,6

The most significant observation to emerge from the stripping procedure is that the width of the surface line is $\sim 50 \mathrm{meV}$ wider than the bulk line. Crystal-field splitting cannot explain this result because it broadens only the $p_{3 / 2}$ component, and would yield an oscillating highbinding-energy tail in the stripped spectrum, which is not observed. Enhanced lifetime broadening of the $2 p$ surface core levels, which are dominated by core-valencevalence (CVV) Auger transitions, is also not a viable explanation. In W(110), whose valence-band density of states (DOS) is significantly modified at the surface, only a $40 \%$ increase in the CVV Auger decay rate is observed. ${ }^{7}$ Not only would such an effect be even smaller in the alkali metals because their surface DOS are more similar to those of the bulk, but the $2 p$ lifetime width of bulk $\mathrm{Na}$ is only $10.15 \pm 1 \mathrm{meV},{ }^{8}$ clearly too small to account for the large observed difference. An increased singularity index $\alpha$ at the surface would make the line more asymmetrical, but would not affect its peak. Analysis of our data as a function of photon energy shows only minor variation of $\alpha$ with relative surface strength, confirming a recent theoretical calculation ${ }^{9}$ which predicts identical $\alpha$ 's for bulk and surface $\mathrm{Na}$.

This leaves inhomogeneous and/or phonon broadening as possible sources of the extra surface width. Both are expected to produce Gaussian contributions to the line shape. In order to establish that the extra surface width is indeed Gaussian requires least-squares analysis of the data. Figure 2 shows the result of such a fit to the data from Fig. 1. Averaged over all measured photon energies for $77-\mathrm{K}$ samples, the surface peaks are characterized by an extra Gaussian width of $92 \pm 12 \mathrm{meV}$ (added in quadrature to the bulk Gaussian width). The spinorbit parameters obtained from the stripping procedure are reaffirmed by the fits. In addition, we obtain a singularity index $\alpha$ of $0.190 \pm 0.016$, in good agreement with an earlier value of $0.198 \pm 0.015,{ }^{3}$ and a Lorentzian lifetime width of $13 \pm 3 \mathrm{meV}$, consistent with absorptionand emission-edge results. ${ }^{8}$

To distinguish between inhomogeneous and phonon broadening, we have performed temperature-dependent measurements of the $2 p$ photoemission. Figure 3 shows spin-orbit-stripped and smoothed spectra obtained at three different temperatures. The increasing width with temperature of both the bulk and surface components is apparent. Moreover, the surface width increases at a faster rate than the bulk as seen by the decrease of the peak height of the surface line relative to the bulk. (A shift in binding energy with temperature due to expansion of the lattice ${ }^{3}$ is also evident.)

A guide to quantitative analysis of the extra surface width versus temperature is obtained by considering the coupling of the core-hole excitation to a longitudinal Debye phonon spectrum. Such coupling, first studied by Overhauser $^{10}$ and later refined by Hedin and Rosengren, " expresses the core-hole excitation of phonons by a Gaussian broadening whose FWHM value, $G_{\text {el-ph }}$, at

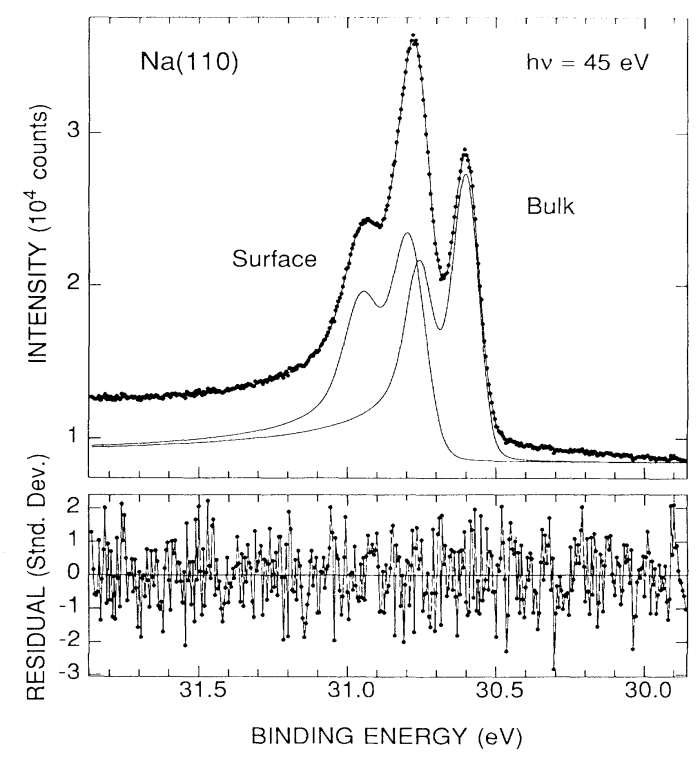

FIG. 2. Least-squares analysis of data from Fig. 1. Surface spectrum is Gaussian-broadened version of bulk spectrum. Note only statistical deviations in residuals. 


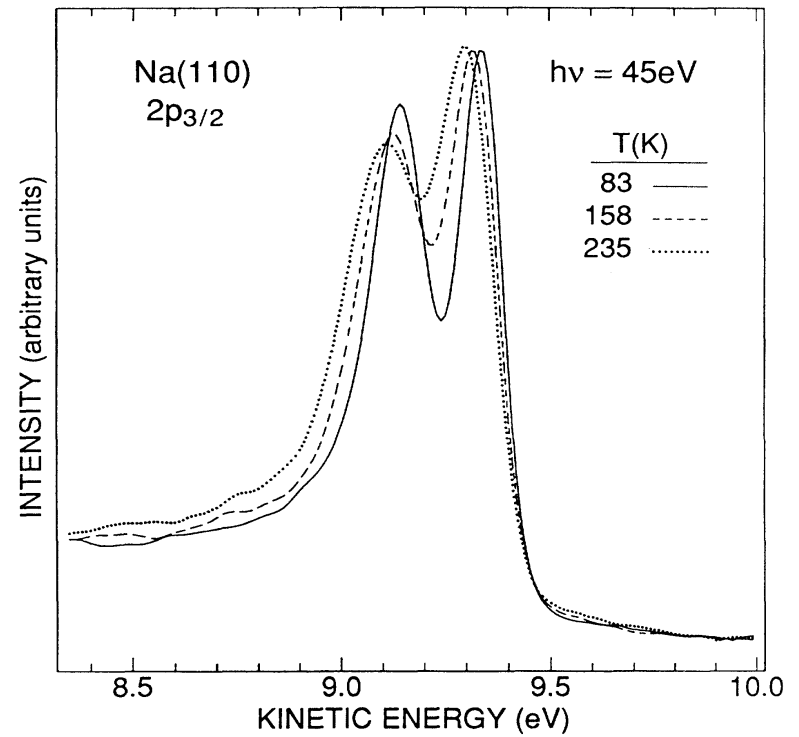

FIG. 3. Smoothed and spin-orbit-stripped $2 p_{3 / 2}$ data at three temperatures normalized to the height of the bulk component.

temperature $T$ is given by

$$
G_{\mathrm{el}-\mathrm{ph}}^{2}=\frac{C}{\Theta_{D}}\left[1+\left(\frac{8}{3} \frac{T}{\Theta_{D}}\right)^{2}\right]^{1 / 2} .
$$

Here, $\Theta_{D}$ is the Debye temperature and $C$ is a parameter which depends upon the valence-band structure and mass density of the solid. Since contributions to the total measured Gaussian width $G_{T}$ add in quadrature, we may write

$G_{T}^{2}=G_{\mathrm{res}}^{2}+G_{\mathrm{inh}}^{2}+C \frac{8}{3} \frac{T}{\Theta_{D}^{2}}\left[1+\frac{1}{2}\left(\frac{3}{8} \frac{\Theta_{D}}{T}\right)^{2}+\cdots\right]$,

where $G_{\text {res }}$ and $G_{\text {inh }}$ are widths from the instrumental resolution and inhomogeneous broadening, respectively. The square of the phonon width has been expanded for large $\left(T / \Theta_{D}\right)^{2}$. Note that in this limit (actually for $\left.T / \Theta_{D}>0.8\right)$, the phonon contribution is very linear in temperature and that the zero-temperature extrapolation of this high-temperature linear regime simply yields the sum of the instrumental and inhomogeneous contributions.

Using a least-squares analysis of the temperaturedependent data, values of $G_{T}^{2}$ for the surface and bulk lines between 77 and $250 \mathrm{~K}$ have been extracted and plotted in Fig. 4. Linear fits to the bulk data above 125 $\mathrm{K}\left[\Theta_{D} \sim 150 \mathrm{~K}\right.$ for $\mathrm{Na}$ (Ref. 12)] and to all of the surface data are also shown. Most notable is the large difference in slope between the two curves; the square of the surface width rises $2.6 \pm 0.3$ times faster than that of the bulk. The linearity at the highest temperatures indi-

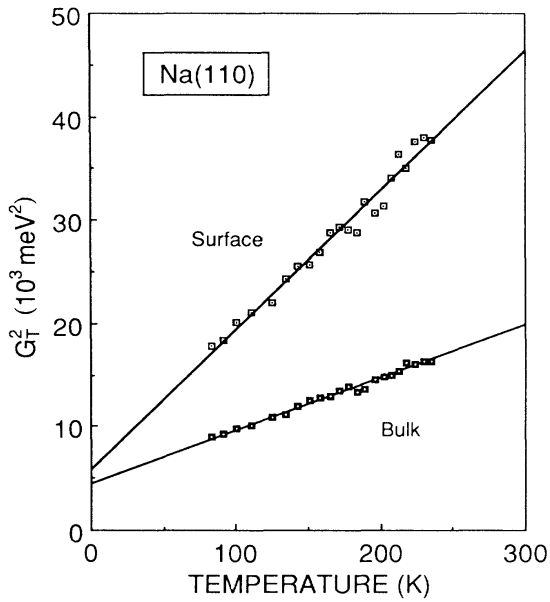

FIG. 4. Square of the total Gaussian width vs temperature for $2 p$ bulk and surface components. Note very different temperature dependence of surface line. Both least-squares-fit straight lines extrapolate to the square of the instrumental width.

cates that anharmonic effects are not important, even at the surface. Both linear extrapolations cross the zerotemperature axis at nearly the same point. Significantly, this point is close to the resolution determined from the Fermi-edge cutoff, thereby confirming the validity of this analysis. The slightly higher value for the surface indicates that a small amount of inhomogeneous broadening may contribute at the surface, but this is negligible compared to the inherent vibrational broadening. From the linear fit we deduce bulk vibrational widths of $71 \pm 6$ $\mathrm{meV}$ at $80 \mathrm{~K},{ }^{13}$ and $124 \pm 10 \mathrm{meV}$ at $300 \mathrm{~K}$. These compare favorably with earlier, less precise determinations of bulk phonon widths in $\mathrm{Na}, 90 \pm 20 \mathrm{meV}$ at $80 \mathrm{~K}$ and $160 \pm 40 \mathrm{meV}$ at $300 \mathrm{~K}^{3}$ By contrast, the surface width is $104 \pm 10 \mathrm{meV}$ at $80 \mathrm{~K}$ and extrapolates to $201 \pm 20 \mathrm{meV}$ at room temperature.

What accounts for the extra surface width? In the bulk, because of symmetry, the vibrational motion accompanying the excitation of a core electron resides solely in the surrounding atoms of the lattice. Considering just the nearest neighbors (NN), their relaxation to a new equilibrium distance around the bulk core-excited atom is constrained by their bonds with other NN's. At the $\mathrm{Na}(110)$ surface, the same is true of relaxations induced parallel to the surface and is reflected in parallel Debye temperatures $\Theta_{\|}$for bcc (110) surfaces which are very close to bulk values. ${ }^{14}$ However, in addition to these NN motions at the surface, the excited atom itself can relax normal to the interface. Because the new equilibrium distance between the excited surface atom and the underlayer atoms is restrained to first order only by relatively weak bond-bending forces between it and other surface atoms, relaxation of surface atoms is far greater than the relaxation of neighboring atoms. This ease of 
motion normal to the surface is reflected in perpendicular Debye temperatures $\Theta_{\perp}$ that are calculated to be much smaller than bulk values ${ }^{14}$ and lead to the enhanced vibrational broadening at the surface.

To express this broadening, Eq. (1) can be easily extended to the surface since $G_{\text {el-ph }}^{2}$ comes from a weighted sum of mean-square displacements of appropriate phonon modes. ${ }^{11}$ For $\left(T / \Theta_{D}\right)^{2} \gg 1$, we obtain

$$
G_{\mathrm{el}-\mathrm{ph}}^{2}=\frac{C}{3} \frac{8}{3}\left(\frac{1}{\Theta_{\|, 1}^{2}}+\frac{1}{\Theta_{\|, 2}^{2}}+\frac{1}{\Theta_{\perp}^{2}}\right) T,
$$

where $\Theta_{\perp}$ is the Debye temperature for phonon modes polarized normal to the surface and $\Theta_{\|, i}$ are the two Debye temperatures for in-plane vibrations. Combining Eqs. (1) and (3) and approximating $\Theta_{\|, i}$ by $\Theta_{D}$, we determine from the data in Fig. 4 that $\Theta_{\perp} / \Theta_{D}$ $=0.41 \pm 0.03$ for $\mathrm{Na}(110)$, provided $C$ is the same in bulk and surface. ${ }^{15}$ This is somewhat larger than the theoretical estimate of 0.32 for $\mathrm{Na}(110),{ }^{14}$ but like that estimate it is well below an average theoretical value of $\sim 0.6$ for a set of five bcc (110) transition-metal surfaces. $^{14}$ Our experimentally determined value of $\Theta_{\perp} / \Theta_{D}=0.41$ for $\mathrm{Na}(110)$ implies that as much as $75 \%$ of the Gaussian width (in quadrature) comes from the excitation of perpendicular modes. Data obtained from $\mathrm{K}(110)$ and $\mathrm{Rb}(110)$ surfaces also yield quantitatively similar behavior. ${ }^{16}$

Since perpendicular Debye temperatures at metal surfaces are, in general, expected to be smaller than the bulk value, it is no surprise that broadened surface lines have been reported for other metals. If Eq. (3) does indeed have general validity, it should account for these other results as well. The reported broadenings have invariably remained uninterpreted because of experimental inadequacies and/or the lack of a suitable theoretical formalism. As an example, bcc (110) transition-metal surface atoms have $\Theta_{\perp} \simeq 0.6 \Theta_{D}$, and so should exhibit [for $\left.\left(T / \Theta_{D}\right)^{2} \gg 1\right]$ phonon widths that are $\sim 30 \%$ larger than atoms in the bulk. This is, in fact, consistent with data obtained on W(110). ${ }^{7}$ For low-index bcc (100) and bcc (111) surfaces, which are calculated to have $\Theta_{\perp}$ and both $\Theta_{\|}$'s of $\simeq 0.6 \Theta_{D},{ }^{14}$ the high-temperature surfacephonon widths are predicted be $\sim 1.65$ times larger than the bulk value. Photoemission data on Ta(111) have been previously analyzed to have room-temperature Gaussian widths of 220 and $320 \mathrm{meV}$ for the bulk and surface atoms, respectively. ${ }^{17,18}$ Subtracting the instrumental resolution in that work of $150 \mathrm{meV}$ yields 160 and $238 \mathrm{meV}$, or a ratio of 1.77 , which is close to our simple estimate of 1.65 .
In summary, enhanced vibrational broadening at a clean metal surface has been observed and characterized for the first time. The phenomenon is dominated by excitation of phonons polarized perpendicular to the metal-vacuum interface. The results of this study should now enable a much more complete and physical interpretation of surface core-level data.

The data were taken at the National Synchrotron Light Source, Brookhaven National Laboratory, which is supported by the U.S. Department of Energy, Division of Materials Sciences and Division of Chemical Sciences.

\footnotetext{
(a) Present address: Department of Physics, The University of Texas, Austin, TX 78712.

${ }^{1}$ See, e.g., D. B. Fitchen, in Physics of Color Centers, edited by W. B. Fowler (Academic, New York, 1968), p. 293.

${ }^{2}$ P. H. Citrin, P. Eisenberger, and D. R. Hamann, Phys. Rev. Lett. 33, 965 (1974).

${ }^{3}$ P. H. Citrin, G. K. Wertheim, and Y. Baer, Phys. Rev. B 16, 4256 (1977).

${ }^{4}$ A. Nilsson and N. Martensson, Phys. Rev. Lett. 63, 1483 (1989); Solid State Commun. 70, 923 (1989).
}

${ }^{5}$ R. Kammerer, J. Barth, F. Gerken, C. Kunz, S. A. Floodstrom, and L. J. Johansson, Phys. Rev. B 26, 3491 (1982).

${ }^{6}$ This last result is not surprising since the (110) surface is the most close packed, and hence most bulklike, of the bcc surfaces. See, e.g., the shifts of low-index surfaces of Ta and W: D. Spanjaard, C. Guillot, M.-C. Desjonqueres, G. Treglia, and J. Lecante, Surf. Sci. Rep. 5, 1 (1985).

${ }^{7}$ D. M. Riffe, G. K. Wertheim, and P. H. Citrin, Phys. Rev. Lett. 63, 1976 (1989).

${ }^{8}$ T. A. Calcott, E. T. Arakawa, and D. L. Ederer, Phys. Rev. B 18, 6622 (1978).

${ }^{9}$ M. Kato, Phys. Rev. B 38, 10915 (1988)

${ }^{10}$ A. H. Overhauser, as reported by L. G. Parratt, Rev. Mod. Phys. 31, 616 (1959), and A. J. McAlister, Phys. Rev. 186, 595 (1969).

${ }^{11}$ L. Hedin and A. Rosengren, J. Phys. F 7, 1339 (1977).

${ }^{12}$ N. F. Mott and H. Jones, The Theory of the Properties of Metals and Alloys (Dover, New York, 1958), p. 14.

${ }^{13}$ This value is obtained from the linear-fit value $(64 \pm 5$ $\mathrm{meV})$ using the $\left(\Theta_{D} / T\right)^{2}$ term in Eq. (2) with $\Theta_{D}=150 \mathrm{~K}$.

${ }^{14}$ D. P. Jackson, Surf. Sci. 43, 431 (1974).

${ }^{15}$ To test whether $C$ is the same for bulk and surface would require data extending to $T \sim \Theta_{D} / 10$.

${ }^{16}$ D. M. Riffe, G. K. Wertheim, and P. H. Citrin (unpublished).

${ }^{17}$ J. F. van der Veen, F. J. Himpsel, and D. E. Eastman, Phys Rev. B 25, 7388 (1982).

${ }^{18}$ G. K. Wertheim, P. H. Citrin, and J. F. van der Veen, Phys. Rev. B 30, 4343 (1984). 